\title{
LAS QUIEBRAS DE LA IDENTIDAD: LA DOBLE FAZ DEL ESPACIO PUBLICO
}

\section{Gabriel Gatti Casal de Rey e Iñaki Martínez de Albéniz} Universidad del País Vasco

\begin{abstract}
RESUMEN
En el País Vasco, tanto las coordenadas que orientan los discursos sobre la identidad colectiva como los escenarios en los que estos discursos se producen y reelaboran han pasado y pasan, tras el proceso de institucionalización política, por una seria redefinición. La emergencia de estas nuevas formas de construir identidad hace necesaria la reelaboración del modelo teórico-metodológico que la sociología ha aplicado a su estudio. Dos momentos estructuran nuestro recorrido: 1) identificar los postulados que soportan este modelo (la socialización entendida como continuidad, los espacios de interacción entendidos como lugares antropológicos, la identidad entendida como actualización de un código que preexiste al sujeto); 2) edificar una óptica alternativa, que encuentra en la imagen de la ruptura su centro orientador (la ruptura de los trayectos de socialización, la crisis que esconde el acceso a los espacios públicos, las quiebras de la identidad). Estos desarrollos se apoyan en el estudio de un caso de especial relevancia sociológica: la gestión que de su entrada a uno de estos escenarios institucionales —el de la Universidad del País Vasco en sus ramas de euskera- hacen sujetos socializados en el posfranquismo.
\end{abstract}

\section{INTRODUCCION ${ }^{1}$}

Vayamos directos al objetivo: este texto nace de una sorpresa, una impotencia, un abandono, y, finalmente, de varias rupturas. Investigábamos un

${ }^{1}$ Este artículo debería ir firmado también por Begoña Abad, pero circunstancias de última hora le han impedido participar en su redacción. De otro lado, la realización de la investigación 
momento de especial significación sociológica, en el que la identidad era puesta en juego, pero, tras los azares del proceso de investigación, nos apercibimos de que el carácter complejo, multidimensional, de la realidad que estudiábamos ponía en juego, desarmaba, la perspectiva de la que partíamos. La puesta en juego de la identidad social nos permitió pasar a jugar con los elementos constituyentes de la identidad de la investigación sociológica ${ }^{2}$.

Estudiábamos la elección de la lengua - euskera o castellano- con la que cursar los estudios en la Universidad del País Vasco. ¿Por qué considerábamos que en la elección se cuestionaba la identidad? Porque imponía la necesidad de optar por un camino y desechar el otro, y, a consecuencia de ello, exigía racionalizar el porqué de esa elección y el porqué de ese rechazo, máxime cuando la lengua es uno de los centros del conflicto simbólico que atraviesa el País Vasco. Supusimos —era lógico- que la socialización primaria marcaría de un modo u otro esos comportamientos. Y así diseñamos el estudio: la elección de rama lingüística constituiría así un dispositivo adecuado para medir el peso de los trayectos de socialización y ver las formas de identidad que éstos comportan.

La existencia de algunas elecciones «anómalas» (la presencia de alumnos que aun habiendo cursado sus estudios básicos y secundarios en ikastolas elegían el castellano como lengua para la Universidad, o de otros que aprendiendo euskera siendo adultos elegían esta lengua) no rompía la correlación entre los antecedentes de socialización y la elección de lengua. Sin embargo, estas elecciones no previstas adquirían un estatuto secundario respecto a las líneas de fuerza del análisis: la potencia de las trayectorias de socialización, el mantenimiento de la identidad. Las incoherencias comportamentales, los discursos ambiguos, los razonamientos difusos, comparecían, necesariamente, como un residuo que en tanto que contradecía o refutaba un núcleo de significados coherentes debía ser desechado en beneficio de la preservación de aquél. El algoritmo es sencillo, de ahí — quizás — el lugar de privilegio que ocupa en las Ciencias Sociales: ajuste/no ajuste de las prácticas y discursos de un sujeto a la matriz que rige lo que se entiende que es «su proceso» normal/modal.

Una serie de postulados soportan esta forma de mirar:

en la que se apoya ha sido posible gracias a la financiación de la Universidad del País Vasco (UPV 002.323-HA 180/94 y UPV 002.323-HA 157/95). Más allá de la obligada mención a las ayudas institucionales, tenemos también que reconocer que esto no se hubiese terminado si no fuese por la ayuda (casi autoría) de Elixabet Imaz, José Mari Iraola, Clarisa Lucas y, en una primera fase, de Mikel Arriaga, que son tan responsables como nosotros del análisis del material empírico y de lo que de éste revirtió en la elaboración del marco teórico de ambas investigaciones.

${ }^{2}$ El valor teórico del hallazgo casual y la potencia que sobre la construcción teórica tienen, en sociología, los encuentros azarosos ya fueron materia de reflexión para Merton (1984: 113), quien, también jugando con la identidad de la investigación sociológica, los etiquetó como serendipity. La resonancia de nuestro trabajo con estas reflexiones de Merton fue una de las muchas observaciones que nos hizo Alfonso Pérez-Agote, a quien agradecemos la atenta lectura del texto. 
1. Postulado de la ubicuidad: que rige la socialización básica de un sujeto y tutela, a su vez, todas sus acciones futuras. Conociendo el núcleo del modelo es factible conocer prácticas, discursos y reacciones del sujeto ante momentos críticos (bifurcaciones, dilemas, crisis y disyuntivas).

2. Postulado de la coherencia: que rige la identidad de un sujeto. El sujeto dispone de un acervo de conocimientos (de unos mapas cognitivos) que le es suministrado desde un núcleo que le preexiste. La identidad es un a priori de la acción.

Frente a éstos, el propósito fundamental es, ahora, el de insinuar y apuntar hacia modos alternativos de reflexión, de manera tal que se evite caer en la tentación de reificar la identidad como modelo que, preexistiendo al sujeto, éste se limita a realizar en lo cotidiano. De evitar caer en eso que Marc Augé ha llamado la "tentación culturalista». Impulsadas por ella, las Ciencias Sociales han buscado actores-tipo que — dice Augé-

"se definirian esencialmente por la cultura de la que cada uno de ellos sería expresión indiferenciada (...). Al fijar y sustancializar indebidamente los conceptos, desvia la atención de los aspectos problemáticos, inestables o dialécticos de la cultura, de las diferencias y tensiones internas de lo social» (1993: 15-16).

La tentación culturalista expulsa al dominio de lo invisible los aspectos problemáticos, las anomalías. Pero éstas revelan la existencia de un orden de realidad inaprehensible desde una forma de mirar regida por lo que Melucci denomina la miopía de lo visible (Melucci, 1989). Una forma de mirar que nos conduce a definir un tercer postulado, el de la centralidad, que rige los criterios que aplica la sociología a lo que analiza. Sus consecuencias son conocidas: localizar un centro - permanente, estable, visible - desde el que el analista pueda ordenar, clasificar y jerarquizar a sujetos, fenómenos y objetos en función de la distancia o lejanía respecto de él.

Estamos argumentando a favor de atender a los núcleos problemáticos, a las rupturas, de atribuir una significación sociológica propia a estos fenómenos aparentemente disonantes. Y esto en un doble sentido: ruptura con los postulados de la ubicuidad y la coherencia; y las rupturas, también, como lugar y momento donde centrar nuestra atención: las fisuras del centro, sus quiebras, comparecen como nuevos campos donde aplicar la mirada sociológica. Es la paradoja de mirar lo social invisible (Barel, 1994: 468) la que se impone. Respecto a ella, Barel indica:

"Por definición, esto es imposible (...). Una paradoja no se suprime, no se supera. Pero se puede intentar dominarla parcialmente, es decir, hacerla habitable rodeándola con astucia. Esto consiste en intentar ver, no lo invisible, sino los procesos de invisibilización, reinstalando la falta de sentido y la proliferación de sentido en el análisis social» (ibidem). 
Para captar las rupturas — los momentos en los que los fenómenos sociales fluyen fuera de la matriz de sentido a la que los conduce concebirlos en términos de coherencia, ubicuidad y centralidad - hemos ideado dos dispositivos de visualización En primer término, considerar la elección en clave de irrupción en el espacio abierto de lo público, de forma que comparecería no tanto como reveladora de predisposiciones cuanto como punto de arranque para la creación de disposiciones con las que orientar la gestión de una situación nueva. De hablar en clave de continuidad, coincidencia y correlación entre antecedentes de socialización y elecciones - como si el camino a seguir en una bifurcación y el sentido del que se dotase a esa elección estuviesen inscritos en un trayecto en curso - a asignar a la elección el carácter de hito (Del Valle, 1995)_-, umbral crítico que deja al sujeto ante una ambigüedad, un reto que se ve obligado a gestionar. Tal reconsideración nos conduce a idear un segundo dispositivo, que atraviesa todo el texto: la óptica de la doble faz. Huiremos así de la tentación culturalista con la que, desde la óptica de la centralidad, son enfocados tanto los espacios institucionales como las identidades que en ellos se desarrollan: elementos que reproducen el centro, componentes del engranaje de un programa del que ambos son su realización

Lo que sigue narra nuestra travesía a través de un objeto de investigación, la elección de rama lingüística, que lejos de su previsibilidad inicial se nos ha mostrado multidimensional: como momento de adaptación a nuevas circunstancias (acceso al espacio público), en el que se desarrollan procesos complejos de circulación carentes de un centro orientador (la sociabilidad institucional), en los que comparecen sujetos competentes para gestionar la ambigüedad y habitar en ella (nueva arquitectura de la identidad). Frente a la ubicuidad, la adaptación; frente a la centralidad, la doble faz; frente a la coherencia, la ambigüedad.

\section{FENOMENOLOGIA DEL LIMITE: RUPTURAS ESPECIALES Y TEMPORALES DE LA IDENTIDAD EN EL ACCESO AL ESPACIO PUBLICO}

Al optar por cursar sus estudios universitarios bien en castellano bien en euskera, el sujeto se ve en la obligación de racionalizar la preferencia por una de las dos alternativas. A través de esa operación parecería disponerse en una posición del espacio social y en una historia comunitaria, una disposición que llevaría, asimismo, implícita una determinada forma de concebir lo que la lengua y la identidad representan ${ }^{3}$. El euskera es, visto desde esta óptica, función

${ }^{3}$ Lo que, a su vez, remite a dos modos opuestos de valorar la lengua: de una parte, aquel que privilegia lo que Pérez-Agote y Tejerina han llamado "representación objetual-simbólica» de la lengua (adhesión al euskera en tanto que símbolo de participación en el ethos comunitario) y, de otra, aquel que considera sobre todo la dimensión instrumental-comunicativa (valoración con arreglo a una lógica pragmática) de la lengua. Acerca de las distintas dimensiones de la lengua, cf. Pérez-Agote y Tejerina (1990). 
de una determinada condición de identidad, más o menos fijada, que la elección de rama hace nítida y visible tanto para el actor como para el observador social: reviste la función de instrumento de diferenciación no tanto entre dos opciones cuanto entre dos grupos sociales.

Y, así vista, la obligatoriedad del paso por la elección de rama naturaliza esa distinción. Con el postulado de la ubicuidad en el horizonte, la Historia (temporalidad que liga el presente con el momento fundacional) y el Territorio de la comunidad (espacio simbólicamente marcado) orientan la decisión del sujeto. En este epígrafe pretendemos extraer la acción de la matriz en la que queda inscrita en esta óptica para pasar a analizar las coordenadas espacio-temporales que le son propias al momento de la elección.

\subsection{La representación de los trayectos biográficos: continuidad y discontinuidad de las trayectorias de socialización}

En los momentos de cruce entre las trayectorias del tiempo personal y del tiempo social, el sujeto es capaz de verse reflexivamente y ordena la secuencia de hechos que constituyen su biografía, organizando sus actividades hasta el presente en una matriz más o menos ordenada. De un modo u otro, se coloca en este momento ante las dos coordenadas del orden social, la espacial y la temporal, ante los componentes cronológico y topológico de ese orden: al verse institucionalmente obligado, en su acceso a la Universidad, a tomar una opción, el estudiante elige una posición y excluye otra, al tiempo que elige un camino y descarta el otro posible.

El valor que los sujetos asignan a este momento crítico en sus trayectorias biográficas puede ser avistado, según Alfred Schütz (1974: passim; Hermida Lazcano, 1995: passim), bien como acto que forma parte de una trama que lo encadena con el pasado del sujeto, bien como una acción que abre un desarrollo de futuro 4 . La ausencia o presencia de causas emplazables en "lo pasado" nos habilita a proponer dos actores-tipo en lo que se refiere a los modos de afrontar la elección: de un lado, aquellos que sitúan ésta en una sucesión de hechos

${ }^{4}$ Para Schütz, dos distintos marcos interpretativos soportan una y otra construcción: 1) Si se hace en relación a referencias — sujetos, objetos, sucesos- situadas en el pasado nos topamos, según Schütz (ibidem: 49-51), con motivos porque: apuntan a lo ya sucedido, a los factores que condujeron hacia una decisión o hacia un acto: son una reconstrucción (operada desde el exterior, sea por el propio actante, sea por un observador) de lo ya realizado. Ajustándose a esta gramática, la continuidad con el trayecto vivido es la matriz en la que el actor sitúa su posición presente y el lugar desde el que la justifica. 2) Si se construye la representación en orden a referencias situadas en lo contemporáneo, nos encontramos, siguiendo a Schütz, ante motivos para: apuntan a lo que puede suceder o sucederá, a los proyectos a los que la acción da paso: son una construcción operada desde el interior que integra referencias situadas en el universo de significados más próximo. La elección es un paso significante de un proceso que se abre, y no pieza o elemento de un proceso ya iniciado. Para una actualización de las categorías analíticas de Alfred Schütz, cf. Hermida Lazcano (1995). 
coherentes, que reconstruye sus motivos ajustándolos a una trama con sentido; de otro, quienes la conciben como momento crítico, dotado de sentido propio y no encuadrable en ninguna trama que estuviese ya preestablecida.

Un modo interesante de formalizar esta discontinuidad lo hemos encontrado en la distinción entre dos formas de concebir los puntos de inflexión en las biografías propias: o bien como elemento integrado en un proceso que ya estaba en curso, o bien como acontecimiento que abre un nuevo proceso y cierra otro (Marzouk-El-Ouriachi, 1991: passim). En función de esto el panorama cobra más claridad, conformándose dos grupos. Por un lado, quienes reconocen el hecho como pieza de una secuencia, como elemento que no afectó a su percepción del mundo. En estos casos se resalta la secuencia sobre el suceso, siendo éste un hecho integrado en una cadena en la que la elección va de suyo. Aquí,

"la reflexión sobre los hitos puede dar lugar a una serie de recuerdos acaecidos con anterioridad a aquello que se reconoce como significativo, a la manera de peldaños que los han ido preparando" (Del Valle, 1995: 285).

Por otro lado, en el sentido contrario, encontramos a quienes, reflexionando sobre su decisión, distinguen la elección como un momento singular en su trayecto biográfico: el suceso es más significativo que la secuencia. La elección es un paso significante de una trayectoria que se abre, y no pieza o elemento de un proceso ya iniciado: las expectativas que el ritual de la elección genera en el actor son los fragmentos con los que elabora un sentido para la acción emprendida. Así, se desbarata para el propio individuo la posibilidad de circunscribir la decisión a una historia, a no ser que sea negándola; es, para él, un acontecimiento disruptor. En este caso, y por oposición al primero,

"la persona tuvo que enfrentarse con una elección que ha afectado el curso de su vida de una manera significativa» (ibidem: 286).

Ciertamente, podrían estos últimos casos ser enfocados como muestras de representaciones negativas de la socialización básica, representaciones negativas que la elección hace visibles para el investigador, todo ello en oposición al primer caso, que ocuparía a los sujetos que se afirman positivamente en lo aprehendido. Pero proceder de este modo supondría reducir la capacidad heurística que hemos encontrado en la distinción entre acontecimiento y elemento, distinción que nos permite pasar de analizar la elección como aparato para el fluir (en forma de motivaciones expresas) de los condicionantes provenientes de los trayectos de socialización de cada cual, a entenderla como dispositivo para interpretar y resolver lo que se presenta como un hito más o menos crucial para el sujeto. Esto se hace especialmente evidente en la segunda de las posiciones (la de quienes ven la elección como acontecimiento), en la que lo relevante es que el conjunto del trayecto biográfico se hace interpretable, visible, y es factible reconstruirlo desde las posibilidades de reflexión que el suceso 
de la elección provee: la elección emerge como un operador de reflexividad. La diferencia entre acontecimiento y elemento traslada, ahora, el análisis de la continuidad de los trayectos a la fuerza de los sucesos.

\subsection{Las representaciones del espacio: la construcción de un espacio valorizado}

El espacio es una categoría de análisis sobre la que se estructura nuestro entendimiento del entorno. Tan es así que buena parte de la terminología sociológica se construye acudiendo a conceptos que, directamente o por analogía, proceden de este terreno: nuestras distinciones fundamentales se apoyan en él, y, también en él, se despliegan nuestras acciones. Por eso, la construcción de mapas mentales, de representaciones topológicas (Moles-Rohmer, 1981: 9), la construcción de un espacio valorizado es un soporte clave de nuestras representaciones del mundo. A esta construcción vamos a atender en lo que sigue, considerando - análogamente a como lo hemos hecho en el epígrafe anterior- que en el momento de entrada a la Universidad el alumnado se ve obligado a elaborar una cierta imagen de lo que significa aquel espacio al que va acceder.

La primera cuestión que es necesario considerar al interesarse por los procesos de construcción de estos mapas mentales es lo que podría llamarse el dominio de existencia que los diferentes escenarios alcancen en el imaginario de un sujeto. Dicho de otro modo, es clave para la edificación de estos mapas que cualquier entidad se distinga en relación al continuo espacio o tiempo en el que está enclavada: una institución o una organización sólo lo será si es representada como una discontinuidad en el continuo de la sociabilidad y si esa discontinuidad se afianza en el imaginario, si se naturaliza y se dota de una cierta duración a lo que la distingue del entorno (Bronstein, Gaillard y Piscitelli, 1995: passim)5.

En el momento del acceso a la Universidad esta discontinuidad está especialmente marcada: la obligación de pasar por distintas fases (selectividad, elección de rama, matriculación), que van desgranando progresivamente el significado del tránsito del alumno desde los lugares de los que procede a aquellos otros a los que accede, le coloca ante una línea, una frontera que separa con nitidez los lugares de su cotidianidad de los ámbitos en los que se va a desenvolver en su futuro inmediato. En el modo de percibir esa diferencia - y, por extensión, de percibir la autonomía de aquello a lo que accede- entran a jugar diferentes sistemas categoriales, distintos cuerpos de valores que orientarán los movimientos (desplazamientos, trayectos) y las expectativas sobre las interacciones que el o la alumna desplegará en su interior.

${ }_{5}$ En el mismo sentido que estos tres autores, A. J. Greimas apunta:

"Un lugar cualquiera sólo puede ser captado fijándolo en relación a otro lugar, el cual no se define sino por lo que no es (...), lo cual lleva consigo que la apropiación de una topía sólo es posible postulando una heterotopia: es tan sólo a partir de ese momento cómo puede instituirse un discurso sobre el espacio" (1980: 142). 
La variabilidad en la atribución de autonomía a estos espacios novedosos es muy amplia: espacio público/privado, espacio propio/ajeno, espacio conocido/extraño... son algunas de entre tantas posibles distinciones. Con cualquiera de ellas podríamos desarrollar el análisis. Ahora bien, la continuidad o discontinuidad que se establezca entre los espacios de la socialización básica y el espacio de la Universidad es la distinción que emerge con más fuerza. Acudimos, para cifrar esta lógica diferencial, a la oposición que el antropólogo francés Marc Augé ha establecido entre los lugares y los no-lugares. Mientras que los primeros se caracterizan por constituir un

"lugar de la identidad (en el sentido de que cierto número de individuos pueden reconocerse en él y definirse en virtud de él), de relación (en el sentido de que cierto número de individuos, siempre los mismos, pueden entender en él la relación que los une a los otros) y de historia (en el sentido de que los ocupantes del lugar pueden encontrar en él los diversos trazos de antiguos edificios y establecimientos, el signo de una filiación)» (1994: 147),

los no-lugares comparecen como su contrario lógico. En palabras de Augé:

«Si un lugar puede definirse como lugar de identidad, relacional e histórico, un espacio que no pueda definirse ni como espacio de identidad ni como relacional ni como histórico, definirá un no lugar» (ibidem: 83).

En efecto, el espacio universitario presenta una textura distinta respecto a los lugares de la socialización, alterando el trayecto que marca la socialización primaria y señalando una cierta distancia respecto de los lugares en los que se produjo De hecho, el término lugar, al menos en el sentido aquí empleado, está inscrito en una lógica que lo hace privativo de los espacios de la socialización primaria. Es así como, en función de la calificación del espacio universitario como lugar (espacio con una marca de identidad clara para el sujeto) o como no-lugar (espacio sin marca de identidad evidente), surgirán representaciones distintas y será diferente la intensidad con la que se demarque la línea que lo separa del área circundante. Con una u otra de soporte, el espacio universitario puede ser integrado en una representación que es inteligible para el actor, en un sistema clasificatorio con el que orientará las prácticas que vaya a desempeñar en su interior. Mantendrá intacto o reconstruirá su mapa topológico, para, en definitiva, dotar de contenido a aquello a lo que accede y a las prácticas que en ese escenario novedoso desplegará. Los contenidos que se asocian a cada extremo del par lugar/no-lugar orientan, pues, la primera percepción del espacio. Desde la observación de los modos de representar el límite que separa lo que queda atrás de lo que viene iremos adentrándonos en la estructura interna de los mapas topológicos que los estudiantes construyen ${ }^{6}$.

${ }^{6}$ Sintéticamente, puede decirse que en un primer tipo de representación se atiende a una valorización del espacio universitario que expresa el deseo de continuidad respecto a los lugares 
$\mathrm{Al}$ analizar los modos de afrontar el acceso al espacio universitario emerge la primera quiebra de la identidad: la de las trayectorias biográficas. Los modos de representar este momento crítico nos permiten elaborar un esbozo de las coordenadas en las que se enmarca la fenomenología del límite que separa el espacio público de los lugares de la socialización ${ }^{7}$, cuestión fundamental, pues la atribución de un significado, la construcción de una representación del dentro y del fuera, de lo que los separa y de lo que los une, se va a conformar en la matriz con la que se elaborarán, a posteriori, las estrategias a desempeñar dentro del espacio público.

\section{"Hablar de rito de institución es indicar que todo rito tiende a consagrar o a legitimar, es decir, a desestimar en tanto que arbitrario y a reconocer en tanto que legitimo, natural, un limite arbitrario" (1992: 114).}

Sin embargo, en el momento en que unos y otros, actores y observadores sociales $^{8}$, hemos empezado a mirar a lo que la elección daba paso, la distinción

antropológicos: la Universidad comparece como ámbito cognoscible y aprehensible mediante el acervo del que se dispone, como un continuum respecto a los espacios de socialización. Los sujetos que se acogen a este primer tipo de representación intentan capturar el significado del espacio público valiéndose del stock de conocimientos disponible: se evoca el aprendizaje básico realizado en las instituciones educativas que preceden a la Universidad. En el extremo opuesto, el marco de referencia general desde el que se le asigna un primer sentido al espacio universitario corresponde al campo de lo novedoso: diferentes sujetos, diferentes agentes formales componen la caracterología de lo que se lee en primera instancia como espacio de posibles. Aquí, el carácter inédito de la Universidad se hace comprensible —inversamente al primer caso- en oposición al marco con el se compone un sentido global de lo ya vivido: los distintos elementos (sucesos, objetos, relaciones) se hacen significativos por lo que tienen de diferente en relación a la socialización básica. En este caso, las instituciones educativas por las que el sujeto ha pasado soportan por negación la representación futura de la institución.

7 Puede extrañar la distinción radical que establecemos entre los lugares de la socialización y los espacios de lo público. Ciertamente, podría argumentarse que la socialización primaria se desarrolla también en espacios públicos. Pero al forzar esta distinción tratamos de destacar los aspectos tópico-ideales de cada uno de estos espacios, esto es, la determinación comunitaria de aquellos que corresponden a la socialización primaria frente a otros —entre ellos, la Universidad- de carácter más indefinido y complejo. Además, esta distinción es congruente con una tendencia histórica, detectada entre otros por Georges Balandier, de pérdida de centralidad de los cuadros tradicionales de socialización. Como afirma este último:

"Las sociedades llamadas avanzadas, en un alto grado organizadas, abiertas a la rápida urbanización y al cambio constante, están siempre en crisis de continuidad. Unos medios sociales y espacios nuevos se forman alli, y los individuos van encontrándose cada vez menos ligados unos a otros por relaciones que se creían más "naturales" que coactivas, y por eso los viejos cuadros de socialización pierden su eficacia” (1975: 68).

${ }^{8}$ Tratamos de hacer autorreflexivo el carácter disruptivo que para los propios actores ostenta la entrada en el espacio público. Asumimos conscientemente que el marco desde el que desplegamos nuestra mirada sociológica es, ciertamente, un marco institucional. En este sentido se pregunta Lourau, y nosotros hacemos nuestra esta pregunta: 
que el rito instituía se desdibuja. En otras palabras, se trastoca la diferencia que aparecía naturalizada y era atribuible a la escisión radical entre dos comunidades (lingüísticas y de identidad) separadas. Lo cierto es que, de acogernos a una perspectiva teórica que se dejase seducir por los efectos inmediatamente discriminantes del ritual de institución, podríamos entender toda "contradicción» entre discursos (en el umbral del espacio universitario) y prácticas (ejecutadas en el interior de la institución) como manifestación de una cierta incoherencia o desafección al grupo ${ }^{9}$. Bien al contrario, estas situaciones ponen de manifiesto las insuficiencias de un análisis que se detenga en el límite que la institución designa. Porque, en otras palabras, tanto los sujetos observados como nosotros, en este caso en tanto que observadores, hemos padecido los efectos de la exigencia de racionalizar la elección (por necesidades prácticas en un caso, por necesidades analíticas en el otro) en términos disyuntivos. Al proceder de este modo, unos y otros hemos refrendado una frontera que separa dos áreas fuertes de significación en el espacio social del País Vasco. Hemos dado carta de naturaleza a una representación de la identidad que es correlato evidente de los postulados de la centralidad, la ubicuidad y la coherencia.

¿Quiere esto decir que lo analizado hasta ahora era innecesario? No, pero sí incompleto: hasta ahora hemos atendido a la fenomenología del limite, a las representaciones que emergen del contacto de unos determinados sujetos con unos límites sociales fuertemente marcados. Pero de detenernos ahí nos acogeríamos a un enfoque apenas atento a la dimensión primaria del espacio público, es decir, a lo que en la institución hay de encarnación de un programa (en nuestro caso, el de la construcción de una sociedad bilingü $\mathrm{e}^{10}$ ) y, consecuentemente, a los discursos y representaciones que los actores (provistos de sus respectivos bagajes culturales) elaboran en relación a éste. Desde esta óptica, la elección revelaría únicamente

"el conocimiento del grado de aceptación y legitimación de la actuación de las administraciones públicas (...) a través de las valoraciones y actitudes que los actores sociales manifiestan en sus discursos» (Tejerina, 1992: 293).

"En qué el compromiso institucional del investigador o del experto (...) es, no un obstáculo para el análisis, sino el lugar mismo donde comienza el análisis institucional?» (Lourau, 1991: 99).

9 De hecho, en el curso de la investigación hemos constatado la existencia de sujetos que, a pesar de adscribirse en el momento de la elección a una de las dos representaciones-tipo arriba descritas, adoptan, una vez superan el umbral de acceso a la institución universitaria, estrategias no congruentes con ellas: oscilan entre ambas, las reelaboran tomando piezas de una y otra. De ese modo se transgrede el orden dicotómico y excluyente que, presuntamente, constituía estas representaciones.

${ }_{10}$ Siendo como es la elección de rama una herramienta diseñada en congruencia con un proyecto de sociedad bilingüe, podría interpretarse, en principio, como un dispositivo que obliga al agente a racionalizar su opción en forma de adscripción a alguno de los grupos portadores de los discursos-tipo que hemos descrito páginas más arriba. 
Desde esta perspectiva, no parece tenerse en cuenta la reelaboración que sufren esos bagajes culturales una vez que el límite deja lugar a la superficie que contiene. El propio Georg Simmel, al afirmar que "en su aspecto formal sociológico, el hecho del marco espacial no consiste solamente en la delimitación política» (1982: 658), atisbó esta cualidad del espacio diseñado institucionalmente. Con su ayuda podemos reformular lo que antes hemos llamado "rito de institución» en términos de lo que Isaac Joseph ha denominado el "umbral de la publicación": una frontera que no determina ni reproduce la cesura que separa a dos comunidades, sino que apunta a distinguir dos ámbitos de sociabilidad cualitativamente bien distintos, el espacio propio (el lugar antropológico y sus ámbitos) y el espacio público. En estos términos lo explica el sociólogo francés:

"Los territorios de la interacción son reservas y lugares cercados - geográficos o situacionales - cuya función es privativa. Todo el territorio implica pues una definición negativa (por defecto) de lo que es público y una negociación en el umbral. Se puede llamar publicación a esta negociación» (Joseph, 1988: 27).

En definitiva, si bien la fenomenología del límite conduce a situarse en uno u otro polo discursivo, las prácticas desplegadas en el interior del espacio público subvierten esta lógica constitutiva. $\mathrm{O}$ al menos habilitan su transformación. Se trata entonces de superar una visión restrictiva y unidimensional de esa fina frontera que separa los lugares antropológicos de los espacios de lo público, objetivo para el que proponemos adoptar una concepción compleja, en doble faz, de la institucionalización, que más allá de los discursos que los actores elaboran en el umbral de la publicación atienda a las prácticas que desarrollan y a la redefinición de los discursos a que estas prácticas dan lugar en un marco políticamente diseñado.

\subsection{La doble faz de la institucionalización}

En general, podemos decir que toda institución ejerce sobre la realidad una acción de planificación. En este sentido, elabora distinciones (espaciales, temporales o representacionales) y las proyecta, a modo de programa, sobre una realidad social que trata de transformar en una dirección precisa. Es de aquí de donde deriva la fuerza socializante de la institución, su lógica performativa: en todo proyecto institucional subyace un intento de transformación de la realidad que se materializa en una planificación dirigida a encauzar las prácticas de los sujetos cuando están bajo su influjo.

Esto no significa, empero, que los marcos de acción que la institución habilita deban ser concebidos como meros factores constrictivos de la acción social: los marcos institucionales, a la vez que constriñen, posibilitan la acción 
de los sujetos. La institución presenta, en principio, un aspecto bifronte, una doble naturaleza: es permisiva y represiva a una vez (Lourau, 1991: 133); limita el campo en el que el sujeto desarrolla sus acciones, al tiempo que posibilita acciones virtualmente imposibles de ser acometidas si no existiesen espacios reglamentados con base en criterios de acceso generalizado, sin restricciones. La escuela francesa del Análisis Institucional ha sido la que, a través, entre otros, de René Lourau, ha abordado de forma más perspicaz esta dialéctica en doble faz de la institucionalización: la institución es la resultante de la interacción compleja entre lo instituido (la dimensión primaria o "programática») y lo instituyente (la gestión o consumo reflexivo que de aquélla realizan los sujetos).

Lo que realmente nos interesa de la propuesta teórica de Lourau es que nos muestra la institución como la intersección de un programa (en la acepción política-fuerte del término), una acción social, que es su negación, y las formas singulares de interacción que emergen entre ambas. Como señala Lourau,

"asimilada a la única instancia de lo instituido, la institución carece del dinamismo que le confiere la instancia complementaria y opuesta de lo instituyente (...). La unidad negativa de las formas sociales se olvida a favor de un positivismo ciego" (1974: 247).

Atendiendo a esta propuesta, podemos hablar de la existencia de una primera dimensión de la institucionalización que remite a la incidencia que ésta, en tanto que programa, tiene en la realidad. La vertiente compleja, a la que por oposición a la anterior llamaremos secundaria, se caracteriza por la relación dialéctica que se establece entre aquel primer trazo o diseño y la gestión que de los marcos habilitados por la institución llevan a cabo los sujetos, momento en que el programa institucional queda sometido a la negatividad (ibidem) de las relaciones sociales que se desarrollan en su interior.

Para ilustrar la vertiente compleja de la institución, resulta oportuno acudir a un estudio de caso. Nos referimos al estudio etnográfico que Paul Willis ha desarrollado en su Learning to Labour. Willis parte, como principio rector de su investigación, de una premisa coincidente con el bosquejo teórico de Lourau:

"las instituciones no pueden ser estudiadas como unidades simples. Al menos tienen tres niveles a los que podemos denominar oficial, pragmático y cultural»(Willis, 1988: 208).

El nivel oficial se refiere a los propósitos formales que la institución tiene con relación a los rasgos estructurales y organizacionales de la sociedad. Es un nivel directamente relacionado con la esfera de lo político y con los determinantes e intereses que operan en este ámbito. El nivel cultural, por contra, alude al depósito cultural propio de los sujetos que conviven en la ins- 
titución ${ }^{11}$. Ahora bien, lo más significativo es que, lejos de estar enteramente determinado por el nivel político, este depósito cultural opera como elemento orientador de las estrategias de adaptación que los sujetos elaboran en su tránsito por la institución. El mundo cultural goza, en consecuencia, de un cierto grado de autonomía, que empuja a los actores sociales a elaborar determinadas estrategias para enfrentarse a la institución, frustrando así las funciones socializadoras de ésta ${ }^{12}$. La conclusión teórica a la que llega Willis trasciende el nivel meramente funcional y termina acogiéndose a una visión de la institucionalización

"que desaconseja cualquier noción simple de causación mecanicista y concede a los agentes sociales implicados alguna perspectiva razonable para contemplar, vivir y construir su propio mundo de un modo que es reconociblemente humano y no reduccionista desde el punto de vista teórico" (ibidem: 201).

No nos interesa tanto el caso estudiado por Willis cuanto las consecuencias teóricas que podemos extraer del mismo. La principal es, tal y como señala Giddens, que todo constreñimiento institucional ha de contar, necesariamente, con la participación activa de los agentes interesados: el programa institucional no se constituye en una fuerza de la que los sometidos a él fueran receptores pasivos (Giddens, 1995: 315) ${ }^{13}$. Es así que, en el caso estudiado por Willis, la contracultura de los estudiantes y sus manifestaciones más palmarias (la burla, el sarcasmo, la risa y otras estrategias que adoptan ante la pretendida «seriedad» de la escuela), lejos de poder ser analizadas en términos de "tendencias

${ }^{11}$ Prescindimos del nivel pragmático (que en el estudio de Willis se refiere a las estrategias pragmáticas que los agentes de la institución, en calidad de sujetos mediadores, elaboran a la hora de interceder entre la institución y sus clientes) puesto que los dos restantes, el oficial y el cultural, dan medida suficiente de la concepción compleja de institucionalización de la que partimos en este texto.

${ }^{12} \mathrm{La}$ institución analizada por este sociólogo inglés, las llamadas Comprehensive Schools, tiene como objetivo (programa) que los alumnos que provienen de la clase obrera se libren de desempeñar la clase de trabajos que desarrollan sus padres. Tal como relata el autor, los «chicos» terminan, no obstante, desarrollando una contracultura obrera con la que enfrentarse a la escuela, lo que les induce a abandonarla y los destina, finalmente, a ejercer profesiones de "clase». La puesta en práctica del programa institucional termina, pues, obteniendo como resultado aquello que trataba de evitar.

${ }_{13}$ Se pueden dividir en dos las críticas que Giddens hace de aquellos planteamientos, como el funcionalista, que privilegian lo instituido frente a lo instituyente. En primer lugar, una crítica de alcance macrosociológico: los planteamientos funcionalistas no toman en cuenta las consecuencias no deseadas de la acción e invierten la relación fáctica entre acción y consecuencia en el sentido de elaborar lo que Cohen ha denominado «leyes de consecuencia» [Karl Marx's Theory of History, Oxford, Clarendon Press, 1978; cit. en Giddens (1995: 320)]: cada vez que un ítem social dado es funcional para otro, se comprueba que el primer ítem social existe. De otra parte, se realiza una crítica a nivel micro sobre la concepción de actor de la que parte el funcionalismo: el actor sobresocializado, mero reproductor del programa institucional. 
desviadas", han de ser necesariamente tenidas por "rasgos fundamentales de (una) penetración inteligente del sistema escolar» (ibidem).

Es este tipo de penetración inteligente, esta reflexividad práctica que activan los actores a la hora de enfrentarse a la institución, la que nosotros encontramos en los sujetos que acceden al espacio institucional. Sin embargo, cuando los sistemas sociales se conciben como «objeto social» visible o instancia programadora de la realidad,

"se termina insistiendo en el influjo general de un orden legitimo coordinado normativamente como un determinante o "programador" de la conducta social. Esta perspectiva enmascara el hecho de que los elementos normativos de sistemas sociales son demandas contingentes que han de ser sustentadas y puestas en vigencia a través de la movilización efectiva de sanciones en los contextos de encuentros reales. Sanciones normativas expresan asimetrias estructurales de dominación, y las relaciones de los nominalmente sujetos a ellas pueden ser algo muy diferente de meras expresiones de los compromisos que de esas normas se esperan" (Giddens, 1995: 66).

En el marco de nuestra investigación cobra, pues, una importancia crucial la relación compleja, no sometida a automatismo ninguno, que se establece entre, de una parte, la institucionalización de políticas y el desarrollo de soportes físicos para su consecución y, de otra, la forma en la que los actores sociales los habitan, desarrollando en su interior prácticas que coadyuvan a su transformación. La comprensión total de las condiciones en que los sujetos afrontan estos espacios exige observar la dialéctica entre el centro y la periferia de la institucionalización, entre las lógicas que guían la producción de sentido desde el programa institucional (la dimensión primaria de la institución) y las lógicas que guían la recepción diferencial de ese programa en los ámbitos de sociabilidad habilitados por la institución (lo que denominaremos de inmediato su dimensión secundaria).

\section{LOS SUJETOS: FORMAS DE CONCEBIR Y GESTIONAR LA IDENTIDAD COLECTIVA}

Estamos delante de escenarios con dos rostros: el del programa que guía su fundación como institución (la estrategia desde la que brota), el de los movimientos que se deslizan bajo aquél. La compleja articulación entre ambas dimensiones, soportada por los conceptos de doble orientación (del actor hacia los espacios de lo público) y de doble faz (de los procesos de institucionalización), se remata en este epígrafe. Los sujetos que participan de ese espacio y que se desenvuelven en esa red institucional son sus protagonistas. Nuevamente, la doble faz articula la arquitectura de nuestra reflexión, siendo ahora el de la identidad colectiva el terreno en el que se edifica. 


\subsection{La dimensión secundaria del espacio público}

No proponemos sustituir el análisis de la primera dimensión (el programa) por el de la segunda: aquélla contiene a ésta en tanto que determina los límites en los que se desenvolverá. Pero la segunda es la dimensión en la que la primera se dota de contenido, la dimensión en la que el programa se materializa en prácticas concretas. Una vez rebasado el umbral de la publicación, el espacio institucional emerge como un espacio abierto, practicable. Por oposición a la calidad de umbral o límite que adopta en su vertiente primaria, denominaremos espacio secundario a aquel en el que los actores desarrollarán estrategias con arreglo a las cuales «fabricar» identidad. Como apuntan Remy y Voyé:

"Regido por los mismos códigos que constituyen el espacio primario, el espacio secundario no toma sentido más que en relación a aquel frente al cual es una posibilidad de distanciamiento, de puesta a distancia, una posibilidad de hacer y ser otra cosa (...). Es pues, por excelencia, el lugar donde se forjan las identidades y donde se transforman» (1981: 71; la cursiva es nuestra).

En efecto, en el paso de la vertiente primaria del espacio a la secundaria, se instituye un ámbito en el que se construye identidad. Lo que pretendemos poner de manifiesto es que con el nacimiento de los espacios institucionales emergen modalidades distintas de construir sentido, modalidades que es posible concebir porque este tipo de espacios, la dialéctica que se habilita en su interior, es la condición de posibilidad de su despliegue $e^{14}$. Puede formularse así: el espacio público, en su funcionamiento, designa y hace explícitos los límites (los continentes) de la sociabilidad, consintiendo en su interior el juego alrededor de los contenidos de la identidad.

Espacio público más nuevas formas de identidad. No nos exponemos al riesgo de tomar una posición clara y expresa sobre qué precede a qué: ¿el desarrollo de formas concretas del espacio institucional permite que estas modalidades de identidad nazcan o éstas, mediante procesos concretos, condicionan

${ }^{14}$ En el período que sucede a la institucionalización política, en el País Vasco se asiste al paso de una estrategia de mantenimiento y reproducción de lo "constituyente» de la comunidad nacionalista a otra, bien distinta, que encuentra sus puntales en la extensión hacia nuevos escenarios y en la inclusión de nuevos agentes. En lo que a lo más visible se refiere, así podría enunciarse el cambio que se registra en los mecanismos que soportan las estrategias con las que se define la «vasquidad». Pero en otro terreno, menos perceptible, este proceso abre paso a dinámicas no previsibles para un observador sólo atento a la dimensión más evidente, visible e instituida del cambio. De entrada, porque éste es un cambio que habilita una cierta polisemización de lo que «lo vasco» encarna y evoca: ahora, la representación nacional de lo social, ya instituida, actuando a modo de molde, de límite en orden al que se conforman nuevos y muy variados escenarios de interacción, contiene a múltiples agentes, cada uno de ellos practicante de nuevas y variadas estrategias de adaptación. La dimensión secundaria (creativa y adaptativa) del espacio público nace como lugar de interacción y como terreno de observación. 
el despliegue de aquéllos? Probablemente, ambas cosas. Lo que sí es cierto es que para visualizar estas modalidades de identidad hay que, previamente, haber sabido ver los modos de operar de los territorios de lo institucional: es la claridad de los límites de éstos la que posibilita al observador explorar los modos de operar de aquéllas.

De lo dicho anteriormente, las primeras consecuencias son obvias: el «sujeto-de-la-identidad» no puede ya concebirse como portador de un modelo que le preexiste, sino que, al contrario, emerge un sujeto competente para negociar las coordenadas con las que definir su pertenencia comunitaria. Y, en segundo término, una comunidad esférica, sin fisuras, bien afirmada en y por un relato histórico, en y por una clara definición de los límites y contenidos de sus territorios, se encuentra ante el espectáculo de la proliferación de escenarios que, nacidos del proyecto abierto por una nueva dinámica de extensión e inclusión, facultan a los sujetos que por ellos transitan a distanciarse de las líneas maestras con las que fueron diseñados ${ }^{15}$.

Ese salto dibuja una tenue frontera entre dos formas de concebir lo que significa «identidad». Instalándonos sobre ella trazamos la línea divisoria entre los análisis que sobre este tema hizo una primera sociología (la atenta a responder a los interrogantes que proceden de la pregunta "¿qué es?» y «¿cómo se reproduce?» una identidad, sociología soportada por los postulados de la ubicuidad, la centralidad y la coherencia) y una segunda, por la que apostamos, pendiente de contestar a "¿cómo se construye?», «qué se busca?» y "¿dónde se busca?» la identidad. Es en ese límite donde se diseña el edificio teórico que aquí pretendemos esbozar.

Para ello es necesario un cambio de óptica Por eso, en este epígrafe argumentamos a favor de la salida a escena de un tipo de actor al que, genéricamente, se podría calificar de estratega, reflexivo y competente para adaptarse a escenarios ajenos al "propio». Antes de abordar su fundamentación hemos de recordar cuál es la condición de posibilidad de su enunciación: la transformación que sufren los sujetos al pasar por encima de la frontera que los aleja de los lugares antropológicos (territorios de la continuidad y de la duración), y les abre paso al conjunto de fenómenos y procesos que aquí hemos sintetizado con el rótulo de espacio público. Como hemos visto, en ese impasse los sujetos implicados acuden a discursos y referencias con los que hacer inteligible ese hito en su biografía. Pero una vez que esta fase de racionalización está supera$\mathrm{da}$, una vez que se adentran en un territorio que ya no es propio, los discursos en los que se apoyan estos sujetos — también afectados por los "efectos de marco" - adquieren nuevos significados y se hacen susceptibles de nuevos usos.

15 La Universidad pública en general y su escisión en dos ramas es uno de esos escenarios. Pero hay otros, muchos: un amplio abanico de movimientos sociales, las instituciones de enseñanza y transmisión del euskera, los espacios gestados para la enseñanza de la lengua a los adultos, los lugares de la interacción juvenil... 


\subsection{El escenario: la doble faz de los escenarios de interacción}

Ante el espacio público, lo hemos insinuado más arriba, se produce una suerte de desdoblamiento entre los trayectos de la socialización primaria y las prácticas de los actores en situación, entre el plano de las referencias culturales y el uso que los sujetos hacen de ellas tras el paso por el umbral que marca la frontera entre el lugar antropológico y el espacio público. Desdoblamiento que soporta, a modo de diagnóstico, la conjetura con la que estamos trabajando: el dispositivo técnico que alumbra la arquitectura contemporánea de la identidad colectiva es la orientación en doble faz.

Desarrollando esta conjetura, puede decirse que, aunque un escenario funde su delimitación como tal en el programa de una tradición, de una comunidad fuerte, lo que en él se despliegue no tiene, necesariamente, por qué apuntar a la reproducción de aquélla, pudiendo así caber en él secuencias de acción que remitan a otros marcos de interpretación. Un momento en el que se produce un salto clave a los efectos de una sociología de la identidad colectiva: aquel que viene del paso de una concepción inclusivo/exclusiva a otra, más compleja y porosa, en el que la alteridad se generaliza, se hace interior al sujeto y a los espacios en los que se desempeña. Es ésta una transformación que afecta a dos elementos, ambos fundamentales en el desarrollo de nuestra investigación:

1) Por un lado, el actor sale de los lugares antropológicos, protectores $y$ protegidos, para sumergirse en territorios de interacción, en los que, en palabras de Augé, vive la paradoja de espacios de comunicación en los que "nadie está en su propio terreno, pero tampoco en el terreno de otros" (Augé, 1993: 28). Podría decirse que pasa de ordenar su imaginario en términos de un único cuerpo de referencias a situarse en ámbitos en los que asiste al desconcierto de la "multiplicación de las referencias imaginadas e imaginarias» (Augé, 1994: 42).

2) Por otro lado, los sistemas de clasificación de la noción de pertenencia se trastocan: hablar de escenarios y de estrategias supone la disolución de aquella representación del sujeto construida bajo el auspicio de los postulados de la centralidad, la ubicuidad y la coherencia. Pasamos de un actor social que es réplica de un modelo que le preexiste y que encarna la forma auténtica (central u original) de una identidad a otro que, discurriendo por distintos territorios de sociabilidad, desempeña en función de ellos estrategias pragmáticas, estrategias desde las que define y gestiona su identidad en términos de las posiciones que (provisionalmente) ocupa en ese espacio y en ese tiempo social.

\subsection{Las estrategias: identidad, adaptación}

El de estrategia es el concepto que abre paso a la redefinición de la arquitectura de la identidad colectiva que en este artículo postulamos. En lo funda- 
mental, puede definirse así: los agentes disponen de unos recursos que despliegan en función de las características de la situación, activando todos, parte o ninguno de los elementos que componen ese capital hasta poder confeccionar una imagen coherente de si con la que conocer y ser reconocido en ese territorio de interacción. Aunque las piezas con las que se elaboran esas estrategias sean distintas, porque diferentes son también los recursos de los que pueden disponer, lo que interesa resaltar es que el escenario (los efectos de marco) exige elaborarlas ${ }^{16}$. El actor, aun y cuando se apoya en un programa de identidad, no opera como mero agente reproductor de unas pautas de conducta, como agente que pone en práctica unas recetas, sino que se distancia de éstas y hace de ellas un uso adaptado a la situación. Cristina Peña-Marín, en un pasaje en el que resuenan las propuestas teóricas goffmanianas, delinea esta circunstancia con gran claridad:

"El yo que proyectamos en una situación nos produce en ocasiones la sensación de actuar una réplica perfecta de un modelo previamente existente. Pero los sistemas de códigos de comportamiento, de comprensión y de valoración son más puntos de referencia que constricciones inamovibles. Varian, se construyen, se disputan y negocian en el curso de la comunicación» (1986: 56).

Metodológicamente, no se pueden dejar de tener presentes no sólo las características concretas de cada escenario de interacción, sino también la

${ }^{16}$ En el ámbito en el que hemos desarrollado el estudio del que parte este artículo hemos podido distinguir tres estrategias de adaptación al espacio público, soporte de las prácticas con las que los sujetos construyen identidad en esos escenarios. Lo que las diferencia es el grado de porosidad que se atribuya a la frontera que separa los lugares antropológicos de los ámbitos institucionales.

\begin{tabular}{|c|c|c|c|c|}
\hline $\begin{array}{l}\text { Estrategia } \\
\text { conservativa }\end{array}$ & \multicolumn{2}{|c|}{$\begin{array}{l}\text { Estrategia } \\
\text { oscilatoria }\end{array}$} & \multicolumn{2}{|c|}{$\begin{array}{c}\text { Estrategia } \\
\text { transformativa }\end{array}$} \\
\hline$S_{0}\left(S_{1}\right)$ & $S_{0}$ & $\mathrm{~T}_{1}$ & $\left(S_{0}\right)$ & $S_{1}$ \\
\hline $\mathrm{T}_{0}\left(\mathrm{~T}_{1}\right)$ & $\mathrm{T}_{0}$ & $S_{1}$ & $\left(\mathrm{~T}_{0}\right)$ & $\mathrm{T}_{1}$ \\
\hline $\begin{array}{l}\text { traslado al espacio público } \\
\text { de las dinámicas del lugar } \\
\text { antropológico }\end{array}$ & \multicolumn{2}{|c|}{$\begin{array}{l}\text { ajuste fluctuante a distintos } \\
\text { marcos significativos }\end{array}$} & \multicolumn{2}{|c|}{$\begin{array}{c}\text { afirmación en el espacio } \\
\text { público para construir } \\
\text { identidad }\end{array}$} \\
\hline
\end{tabular}

$\mathrm{S}_{0} / \mathrm{T}_{0}$ : espacio/tiempo previo al acceso al espacio público.

$S_{1} / T_{1}$ : espacio/tiempo de la interacción en el ámbito institucional.

En el primer caso, la frontera se diluye: el sujeto habita el espacio público recurriendo a elementos que toma del lugar antropológico. En el tercero, por el contrario, es la consistencia del umbral la que faculta a los sujetos a desarrollar formas de identidad propias de esos escenarios. Por último, en el segundo caso, el umbral presenta un grado máximo de porosidad: distingue dos dinámicas entre las que el sujeto pendula, claramente diferenciadas e igualmente significativas. 
forma en la que el sujeto se lo representa. La referencia a las coordenadas espacio-temporales de la identidad se hace inevitable, pues el sujeto del que hablamos adquiere estatuto de tal solamente en orden a la situación en la que se desenvuelve y sólo se construye como tal por y en las transformaciones y reinterpretaciones que aplica a lo largo de y sobre su propio curso biográfico. Transformaciones y reinterpretaciones que, sintéticamente, pueden concebirse en función de dos ejes:

1) Temporal: donde se acrecientan los momentos de cruce entre el «flujo temporal interno" del actor social con el tiempo exterior, el tiempo social: intervenimos en los acontecimientos del mundo y, a su vez, los acontecimientos del mundo nos moldean como sujetos, instándonos a integrar en una sola duración la pluridimensionalidad de cursos temporales por los que circulamos. Pasamos de la permanencia de significados y la continuidad a la multiplicación de situaciones y roles asumidos:

"Desde el ámbito primario, fuente de las primeras y más sólidas creencias del individuo, donde adquiere un mundo de definiciones y, con él, una identidad, los sucesivos encuentros con los otros incorporan nuevos marcos de conocimiento y de creencia y nuevas versiones de su identidad" (Peña-Marín, 1986: 56-57).

2) Espacial: transita por coordenadas que van desde el propio (el dominio de la mismidad radical) al espacio de lo público (dominado por la marca de su diseño institucional y por las fronteras que designan lo que integran y lo que dejan fuera). Este relativo distanciamiento en relación a los lugares de la comunidad, a los lugares sagrados y protegidos, implica una seria modificación en la forma de representarse el espacio: no estamos nunca en el terreno propio, de tal suerte que asistimos al espectáculo de la proliferación de escenarios de interacción, espectáculo que, como señala Marc Augé, revela que

"cada uno (...) se encuentra en la intersección de diversos mundos (...). Los espacios de comunicación (entre esos sujetos) son de geometría variable, de suerte que cada actor construye su identidad frente a los demás pero preservando la autonomía de cada uno de esos espacios» (1995: 159).

\section{A MODO DE CONCLUSION: LAS FIGURAS DE UNA NUEVA ARQUITECTURA DE LA IDENTIDAD COLECTIVA}

Si las definiciones clásicas de la identidad encontraron en distintos dispositivos técnicos una apoyatura (el nosotros afirmado en la historia en la definición esencialista, el cogito racional para la racionalista), localizar una figura análoga para el caso de formas de identidad de suyo paradójicas es harto difí- 
cil. Y más aún si se quiere definir una figura que no "traicione» el grado de complejidad de los procesos que con ella se quieren describir. Es en ese sentido que hemos advertido una nada despreciable capacidad heurística en la ya citada doble faz del proceso de institucionalización, viendo allí el mecanismo que soporta y explica la doble posición del sujeto contemporáneo respecto a los espacios públicos: un primer movimiento de racionalización de los límites; un segundo, de adaptación y de gestión de las sociabilidades que se despliegan tras ellos. Esta doble tendencia hace visibles las estratagemas que aplica el sujeto propio de esta segunda acepción de la identidad, constructiva y procesual. El cuadro que sigue sintetiza lo sustantivo de esta acepción de la identidad, así como lo que la enfrenta a aquella otra que pretendemos abandonar:

\begin{tabular}{|c|c|c|c|}
\hline \multicolumn{2}{|c|}{$\begin{array}{l}\text { Acepción primera de la identidad: } \\
\text { identidad como referencia exterior al sujeto }\end{array}$} & \multicolumn{2}{|c|}{$\begin{array}{l}\text { Acepción segunda de la identidad: } \\
\text { identidad como proceso reconstructivo }\end{array}$} \\
\hline $\begin{array}{l}\text { Dispositivo técnico: } \\
\text { orientación coherente } \\
\text { y continua hacia las } \\
\text { referencias de la iden- } \\
\text { tidad }\end{array}$ & $\begin{array}{l}\text { sujeto coherente: } \\
\text { identificación plena } \\
\text { (central/auténtica; } \\
\text { periférica/simulada) } \\
\text { con los contenidos } \\
\text { que componen el } \\
\text { programa de la iden- } \\
\text { tidad colectiva }\end{array}$ & $\begin{array}{l}\text { sujeto fragmentario: } \\
\text { identificación lateral } \\
\text { y parcial/pragmática } \\
\text { con las formas del } \\
\text { programa de la iden- } \\
\text { tidad colectiva }\end{array}$ & $\begin{array}{l}\text { Dispositivo técnico: } \\
\text { orientación en doble } \\
\text { faz hacia las referen- } \\
\text { cias de la identidad }\end{array}$ \\
\hline $\begin{array}{l}\text { Figuras para la repre- } \\
\text { sentación de los aspectos } \\
\text { imaginarios y organiza- } \\
\text { cionales de la vida } \\
\text { social: } \\
\text { metáforas monistas } \\
\text { (comunidad, indivi- } \\
\text { duo...) }\end{array}$ & $\begin{array}{l}\text { permanencia de sig- } \\
\text { nificados: continui- } \\
\text { dad y estabilidad, } \\
\text { soportes de los meca- } \\
\text { nismos de transmi- } \\
\text { sión y de las agencias } \\
\text { de mediación/sociali- } \\
\text { zación }\end{array}$ & $\begin{array}{l}\text { multiplicación en } \\
\text { tiempo y espacio de } \\
\text { marcos de encuentro } \\
\text { social }\end{array}$ & $\begin{array}{l}\text { Figuras para la Repre- } \\
\text { sentación de los aspectos } \\
\text { imaginarios y organiza- } \\
\text { cionales de la vida } \\
\text { social: } \\
\text { identificaciones para- } \\
\text { dójicas (identidades } \\
\text { sucesivas, hibridación } \\
\text { cultural...) }\end{array}$ \\
\hline
\end{tabular}

Estamos ante un sujeto reflexivo, que, tal y como indica Fernando García Selgas, construye su identidad en

"un proceso constructivo, narrativo y político, realizado mediante la interpretación reflexiva que (...) hace de su propia biografía y con la que viene a sostener marcos generales de sentido" (1995: 504-505).

Hablamos, es evidente, de una reflexividad que no es ontológica y que tampoco pretende argumentar a favor de la salida a escena de un actor racional. Bien al contrario, sostenemos un modelo de reflexividad situacional, y que 
aboga a favor de un sujeto pragmático (es la propia de un actor que busca adaptarse a las distintas situaciones en las que se desenvuelve). Al contrario que en la primera acepción de la identidad colectiva, en ésta que defendemos el sujeto, más que una permanencia (una entidad asentada en la duración y orientada hacia la continuidad), es una permanencia reconstructiva ${ }^{17}$. Podemos, con Cristina Peña-Marín, enunciarlo como sigue:

"Nuestros diversos momentos deben poseer (...) alguna organización, o hemos de ser capaces de interpretarlos como organizados: explicarnos y explicar nuestros actos conforme a alguna razón, construir una historia personal (...) sobre el supuesto de conocernos y controlarnos (...). Es a través de nuestra capacidad para producir estas realizaciones como nos percibimos como un ser único, dotado de una continuidad» (1986: 54)

El espacio público emerge de este modo como un mecanismo de gran potencia a la hora de construir y reconstruir la identidad del sujeto: permite que se distancie de sí mismo y que agregue nuevas significaciones a su identidad. Es en él donde se hace posible eso que Isaac Joseph llama una "sociología de la adaptación" (1988: passim) porque es en ese terreno donde aparece como competente para jugar con los roles, donde aparece como la instancia en que se realiza la síntesis entre dos de sus facetas: la primera, que le define como "agente actuado (servidumbres de roles definidos fuera y al exterior)" (Wagnon, 1990: 54), y la segunda, que le distingue como "agente actor (autónomo)» (ibidem).

Salta a escena un nuevo tipo de agente. Lo que hace necesario desarrollar nuevos procedimientos de observación y aplicar diferentes corrientes de reflexión. Procedimientos y corrientes que han de partir de una máxima: ya no es posible entender la identidad como un a priori de la acción, sino como la acción misma. Más que nombrar un corpus de elementos que el agente ejecuta, podría decirse que «identidad» designa el proceso en el que este corpus se crea y se recrea: «identidad» aparece ahora como un recurso expresivo al que la sociología tiene acceso para dar nombre a la articulación compleja entre las distintas fases que conforman ese proceso. A saber: la génesis y constitución de los límites por medio de los que se clasifican, separan, ordenan, jerarquizan y discriminan objetos, personas y acontecimientos; los mecanismos de reactivación de esas categorías; y, por último y fundamentalmente, los ámbitos en los que esas categorías y los límites que las conforman se negocian, se reinterpretan, se desdibujan y se redefinen.

Todo esto nos conduce a la necesidad de reformular un mapa de la identidad que se había construido apoyándose en el par centro/periferia y en otros conceptos tangentes para dirigirnos hacia otro, conformado en torno a las nociones de circulación y de frontera: la representación de los límites que separan los distintos escenarios de interacción, las reconstrucciones de la propia

17 Aunque extraída de su contexto original, tomamos esta idea de Navarro (1996). 
biografía que exige el paso por cada uno de ellos y el juego reflexivo que se desarrolla en el tránsito por esos escenarios (cómo representarse, cómo posicionarse ante los otros, cómo circular por ese espacio) son los elementos centrales de este mapa. Es el espacio público la clave desde la que se han ido desarrollando distintas rupturas en nuestra investigación: la primera, de la biografía del sujeto; la segunda, de la lógica del lugar antropológico; y la tercera, quizás la fundamental, la de nuestro propio aparataje teórico: la ruptura, puede decirse así, de la tentación culturalista con la que nos acercamos al escenario institucional. Cambia, con todo ello, el objeto fundamental de la observación: del progra$\mathrm{ma} /$ modelo de la identidad (la tradición y el territorio) y de su actualización y mantenimiento, a los escenarios y agente de su construcción y reelaboración.

\section{BIBLIOGRAFIA CITADA}

Augé, M. (1993): «Espacio y alteridad», Revista de Occidente, núm. 140, 13-34.

- (1994): Los no lugares. Espacios del anonimato, Barcelona, Gedisa.

- (1995): Antropología de los mundos contemporáneos, Barcelona, Gedisa.

Balandier, G. (1975): Antropo-Lógicas, Barcelona, Península.

BAREL, Y. (1994): "De la fermeture à l'ouverture, en passant pàr l'autonomie?», en J. P. Dupuy y P. Dumouchel (eds.), L'autoorganisation: De la physique au politique, París, Seuil.

Bronstein, V.; Gaillard, J. C., y Piscitelli, A. (1995): «La organización egoísta. Clausura operacional y redes conversacionales», en J. M. Delgado y J. Gutiérrez (eds.), Métodos y técnicas cualitativas de investigación en Ciencias Sociales, Madrid, Síntesis.

Bourdieu, P. (1992): «Los ritos como actos de institución», en J. Pitt-Rivers y J. G. Peristany (eds.), Honor y gracia, Madrid, Alianza.

Del Valle, T. (1995): «Metodología para la elaboración de la autobiografía», en C. Sanz Rueda (coord.), Invisibilidad y presencia. Seminario Internacional "Género y trayectoria profesional del profesorado universitario", Madrid, Instituto de Investigaciones Feministas de la Universidad Complutense.

García Selgas, F. J. (1995): "Análisis del sentido de la acción: el trasfondo de la intencionalidad», en J. Delgado y J. M. Gutiérrez (eds.), op. cit.

Giddens, A. (1995): La constitución de la sociedad, Buenos Aires, Amorrortu.

Greimas, A. J. (1980): Semiótica y Ciencias Sociales, Madrid, Fragua.

Hermida Lazcano, P. (1995): «Domesticando el futuro: el tiempo en la sociología fenomenológica de Alfred Schütz», comunicación al V Congreso Español de Sociología, inedito.

JosePH, I. (1988:) El transeúnte y el espacio urbano, Barcelona, Gedisa.

LOURAU, R. (1974): «Institución», en J. Duvignaud (ed.), La sociología. Guía alfabética, Madrid, Siglo XXI.

- (1991): El análisis institucional, Buenos Aires, Amorrortu.

Marzouk El-Ouriachi, K. (1991): "Acontecimiento», en Terminología cientifico-social. Aproximación crítica (Anexo), Barcelona, Anthropos-UCM.

Melucci, A. (1989): Nomads of the Present, Londres, Hutchinson.

Merton, R. K. (1984): Teoría y estructuras sociales, México, D.F., FCE.

Moles, A., y Rohmer, E. (1982): Laberynthes du vécu. L'espace, matière des actions, París, Méridiens.

Navarro, P. (1996): «Hacia una teoría de la morfogénesis social», en A. Pérez-Agote e I. Sánchez de la Yncera (eds.), Complejidad y teoría social, Madrid, CIS.

Peña Marín, C. (1986): «La identidad en la frontera con los otros», Revista de Occidente, núm. $56,53-57$. 
Pérez-Agote, A., y Tejerina, B. (1990): «Lengua y actor social: un enfoque teórico de sus relaciones», REIS, núm. 49, 145-159.

Remy, J., y VOYE, L. (1981): Ville, ordre et violence, París, PUF.

SCHÜTZ, A. (1974): Estudios sobre teoría social, Buenos Aires, Amorrortu.

Simmel, G. (1982): Sociología. Ensayos sobre las formas de socialización, Madrid, Alianza.

TEJERINA, B. (1992): Lengua y nacionalismo, Madrid, CIS-Siglo XXI.

WAGNON, C. (1990): «Tout est dans la façon: remarques sur l'identité, les jeux, les rôles», Sociétés, núm. 30, 51-62.

WILLIS, P. (1988): Aprendiendo a trabajar, Madrid, Akal.

\begin{abstract}
In the Basque Country, both the coordinating committees which direct the discourses on the collective identity and the scenarios in which these discourses are held and reworked have been, and continue to be, subject to a profound redefinition in the wake of the political institutionalisation process. The emergence of these new identity-building mechanisms calls for a redefinition of the theoretical-methodological model sociology has applied to their study. Our trajectory is shaped by two specific tasks: 1) identifying the hypotheses that underpin this model (socialisation understood as continuity, interaction spaces understood as anthropological areas, identity taken to mean the updating of a code that precedes the subject);2) developing an alternative viewpoint, which finds in the image of breakdown its guiding centre (the breakdown of socialisation trajectories, the crisis concealed by access to public spaces, identity collapses or crises). These developments are based on the study of a case of particular sociological relevance: the steps by virtue of which access to one of these institutional scenarios - that of the University of the Basque Country in its Euskera branches- generates subjects socialised in the post-Franco era.
\end{abstract}

